\title{
A cross-correlation sub-sampling receiver for low power applications in a low SINR environment
}

\author{
M. R. M. Mikhael, M. S. Oude Alink and A. B. J. Kokkeler \\ University of Twente, The Netherlands
}

\begin{abstract}
Wireless sensor networks have recently emerged in a wide range of applications. Many attributes are essential for such networks such as: low cost, small form-factor, limited peak power consumption and the ability to operate in harsh interference scenarios. Most of these networks do not require high data-rates to operate. In this respect, sub-sampling receivers have shown promising results but suffer from noise folding and interference aliasing. In this paper, a sub-sampling receiver in combination with cross-correlation is used to enhance sensitivity and interference robustness while maintaining the sub-sampling advantages. An architecture which uses two different sampling frequencies is proposed. It shows $\sim 2 \mathrm{~dB}$ SNR improvement compared to traditional architectures due to cross-correlation and an additional $\sim 2 \mathrm{~dB}$ for each doubling of integrations. For a BER of $10^{-3}$, the required SIR is reduced with $4.5 \mathrm{~dB}, 11.5 \mathrm{~dB}$ and 14.5dB after using cross-correlation with the same, half and quarter data-rate used respectively. These improvements allow for a lower-power and lower-cost implementation.
\end{abstract}

Keywords-Ultra-low-power receivers, sub-sampling receiver, cross-correlation, noise-folding reduction, interference robustness.

\section{INTRODUCTION}

Recently, wireless sensor networks (WSNs) have been used in various applications such as building automation, industrial applications, traffic tracking and environmental monitoring. This development was enabled due to the huge advances in wireless communications and digital electronics which were essential to produce low-cost, low-power nodes of WSNs [1].

The specifications of these WSN nodes impose many requirements on the receiver design. The peak power consumption of the receiver should be in the range of a few mWs to allow the usage of small/cheap batteries or energy harvesters. The use of unlicensed ISM bands for wireless communications requires a highly interference robust receiver. Finally, high flexibility is of great importance to many WSN nodes which encourages the usage of flexible digitally-inspired techniques. Using more digital circuits in the receiver also benefits from technology scaling (Moore's law).

In most traditional receiver architectures, such as the direct conversion and low intermediate frequency (IF) architectures, the receiver includes power-hungry blocks such as the RF local oscillator and mixer. The sub-sampling architecture uses a sampling frequency lower than the RF carrier frequency to directly down-convert RF signals to baseband which reduces the receiver power consumption.

Sub-sampling receivers suffer from noise-folding. Since the noise in all the image bands folds into the baseband, the signal-to-noise ratio (SNR) of the received signal is degraded. This degradation is proportional to the ratio between the RF (a)

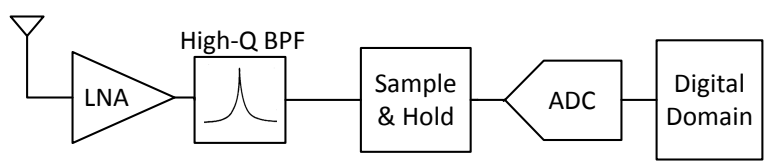

(b)

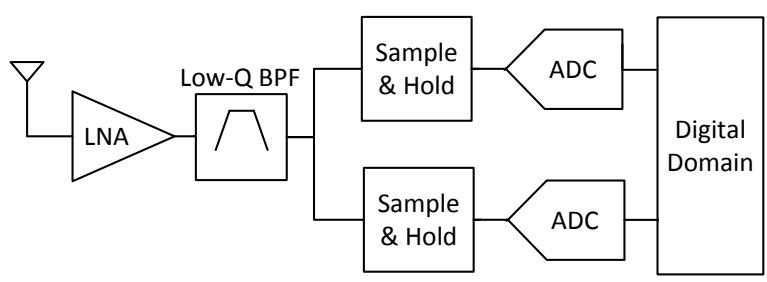

Fig. 1. (a) The traditional sub-sampling receiver where analog components with tough specifications are required for acceptable performance of the receiver. (b) The proposed implementation where the digital domain includes noise reduction and interference cancellation by means of cross-correlation.

noise bandwidth and the quadrature sub-sampling frequency [2]. Like the noise, interfering signals in the image bands will be down-converted to the baseband which degrades the interference robustness of the receiver.

In order to deal with noise and interference aliasing, high quality factor (Q) RF-filtering is typically required. In [3], a fixed filter was proposed for filtering the noise and interferers in the image bands but this filter restricts the flexibility of the receiver. In [4], the RF-filtering was achieved using an offchip flexible BAW-resonator which still would increase the form-factor. The same would be true for SAW-filters. In [5], an on-chip resonant LC-filter was used but it suffers from a low-Q filtering. Another on-chip solution was proposed in [6] in which an enhanced-Q low-noise amplifier (LNA) was used. However, it consumes a lot of power and area, and requires periodic optimizations. Recently, N-path filters have shown great potential for high-Q filtering but they are rather power hungry for our application [7].

In this paper, we propose using cross-correlation (XC) in the digital domain as an alternative to the previous analog solutions. Fig. 1 illustrates the main target of this paper, which is to relax the analog components specifications by exploiting techniques in the digital domain which can lead to savings in area, power and/or design complexity. The proposed architecture will use XC to suppress noise and interference. The architecture was implemented and tested with a lab setup to examine its benefits and trade-offs, and the main results and conclusions are reported. 


\section{CROSS-CORRELATION}

In traditional energy detection based on auto-correlation (AC), only one receiver is used. The average power estimate $P_{\mathrm{AC}}$ is evaluated:

$$
P_{\mathrm{AC}}=\frac{1}{K} \sum_{k=0}^{K-1}|r[k]|^{2}
$$

where $r[k]$ is the complex baseband receiver output and $K$ is the total number of samples. By averaging $K$ samples, the variance of $P_{\mathrm{AC}}$ reduces, which improves the estimate. However, the estimate remains biased due to the noise power in $|r[k]|^{2}$.

Using XC to mitigate noise uncertainty of the receiver was proposed for energy detection applications such as spectrum sensing [8]. In XC, two receiver chains are used and their outputs are correlated with each other to evaluate the average power estimate $P_{\mathrm{XC}}$ :

$$
P_{\mathrm{XC}}=\frac{1}{K} \sum_{k=0}^{K-1} r_{1}[k] r_{2}[k]^{*}
$$

where $r_{1}[k]$ is the complex baseband output of the first chain and $r_{2}[k]^{*}$ is the complex conjugate of the complex baseband output of the second chain.

The same input signal is processed in both chains and the noise at each chain is assumed to be uncorrelated. This can be modeled by the two uncorrelated ${ }^{1}$ noise sources $n_{1}$ and $n_{2}$ in which $E\left[n_{1} n_{2}\right]$ equals zero. The variance of $P_{\mathrm{XC}}$ also decreases with longer averaging. However, unlike for $P_{\mathrm{AC}}$, there is no bias in the estimate for $P_{\mathrm{XC}}$. This, as will be shown later, plays a crucial role in the demodulation of a received signal.

$\mathrm{XC}$ was proposed to achieve different targets:

\section{a. Improve sensitivity/noise figure:}

Decreased noise levels due to XC can directly be translated into lower sensitivity requirements as the noise power after correlation is reduced. As a rule of thumb [8], the sensitivity is improved by an additional $1.5 \mathrm{~dB}$ per doubling of the number of samples to be integrated. If the improvement in sensitivity is not needed, XC can be used to allow using front-ends with higher noise figure which, in return, would mean consuming less power in the receiver.

\section{b. Relax linearity requirements:}

$\mathrm{XC}$ was also proposed to increase the linearity of the receiver in which the receiver can be designed for high linearity instead of low noise. In [9], the receiver was designed with an attenuator at the input of an LNA to enhance linearity where the excess noise was removed by $\mathrm{XC}$.

\section{ness:}

c. Improve harmonic rejection \& interference robustIn [10], two receivers with a small frequency difference between their local oscillator frequencies are used. The resulting harmonic images in the two receivers are uncorrelated since they appear at different frequencies. After XC, the harmonics are suppressed compared to the fundamental signal.

\footnotetext{
${ }^{1}$ In real implementations, $n_{1}$ and $n_{2}$ will not be completely uncorrelated. This correlated contribution may degrade the performance.
}

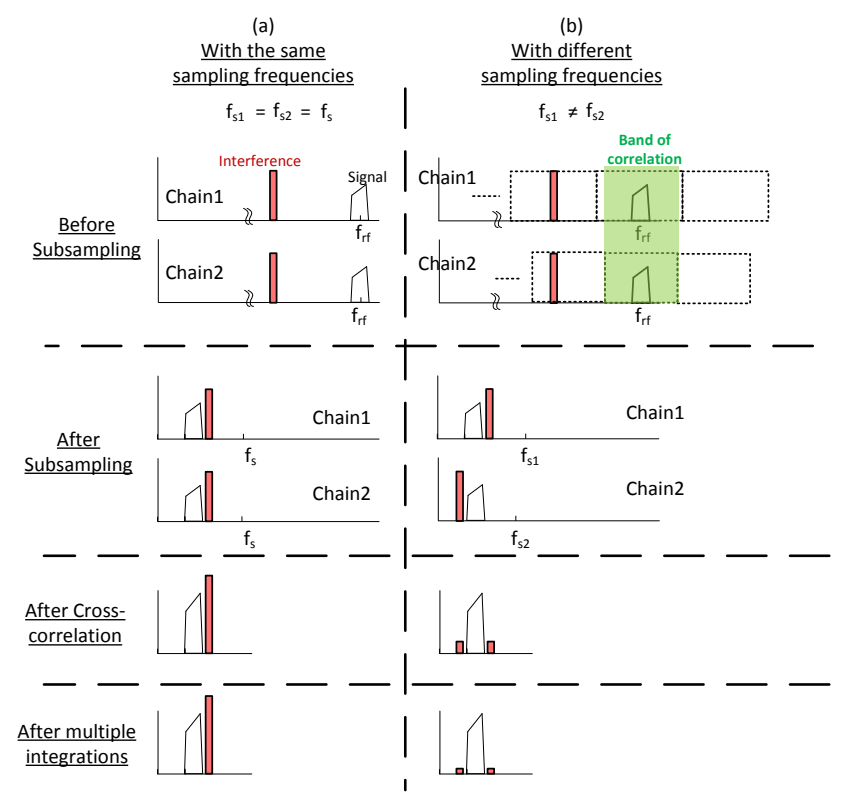

Fig. 2. The frequency response of XC in case of: (a) Same sampling frequencies (interference suppression does not improve) and (b) Different sampling frequencies (interference suppression improves since interferers appear at different frequencies in the two chains).

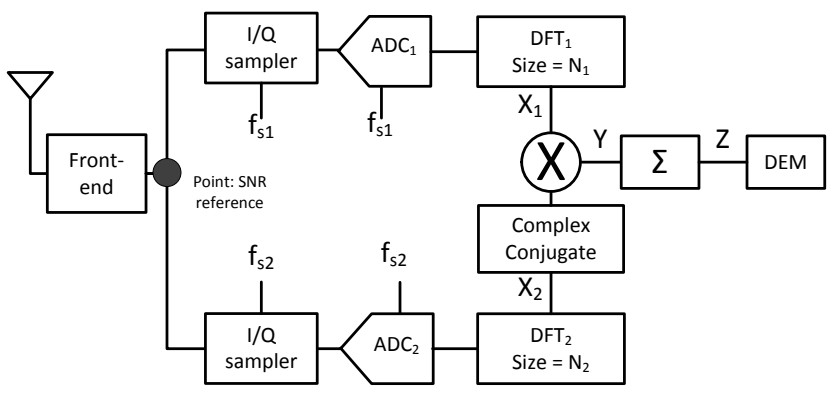

Fig. 3. The proposed implementation using $\mathrm{XC}$ and two receivers chains based on a sub-sampling architecture.

\section{THE SUB-SAMPLING CROSS-CORRELATOR}

Unlike the previously mentioned works that only focused on spectrum analysis, we will exploit $\mathrm{XC}$ while actually demodulating the RF input signal. In order to improve interference robustness of the receiver, we propose to use different coprime sampling frequencies in the receiver chains. Choosing these frequencies carefully allows specific frequency bands in the RF domain to appear at the same frequencies in the digital baseband of both chains. The desired signal should reside in one of those bands which will be called bands of correlation. Meanwhile, any signal outside those bands would appear at different frequencies in the baseband of the two chains as illustrated in Fig. 2. This will ensure that $\mathrm{XC}$ is effective in interference suppression. Using different co-prime sampling frequencies with sub-sampling receivers was proposed before for sparse spectrum sensing [11].

Fig. 3 shows the proposed architecture of the receiver. It begins with the antenna and the analog front-end which includes an LNA and some RF-filtering before the sub-sampling chain. 
The specification of this filter will be relaxed due to the noiseand interference reduction by the $\mathrm{XC}$, and thereby reduces its power consumption. The following stage includes the two receiver chains. Each starts with a sampler which performs I/Q down-conversion, followed by digitization. In the digital domain, $\mathrm{XC}$ and demodulation are performed.

Since the two chains use different sampling frequencies, thus different clocks, combining their outputs in the XC stage requires some attention. We propose to deal with this by executing $\mathrm{XC}$ in the frequency domain. In this case, two DFTs are used with different sizes, $N_{1}$ and $N_{2}$, such that the frequency resolution $f_{\text {res }}$ is the same in both chains:

$$
f_{\text {res }}=\frac{f_{s 1}}{N_{1}}=\frac{f_{s 2}}{N_{2}}
$$

where $f_{s 1}$ and $f_{s 2}$ are the sampling frequencies in the two chains. Assuming $f_{s 1}<f_{s 2}$, the band of correlation can be defined as those frequency ranges where frequency $f$ satisfies the following expression:

$$
m f_{\text {res }} \cdot \operatorname{lcm}\left(N_{1}, N_{2}\right) \leq f \leq m f_{\text {res }} \cdot \operatorname{lcm}\left(N_{1}, N_{2}\right)+f_{s 1}
$$

where $1 \mathrm{~cm}$ is the least common multiplier and $m \in \mathbb{N}$.

Each receiver chain will produce both I and Q outputs. In chain $1, N_{1}$ complex samples of each symbol are used as input for $\mathrm{DFT}_{1}$ in which the I and Q components of the vectors are defined as:

$$
\begin{aligned}
& \bar{i}_{1, k} \triangleq\left[i_{1}\left(k N_{1}\right), i_{1}\left(k N_{1}+1\right), \ldots, i_{1}\left((k+1) \cdot N_{1}-1\right)\right] \\
& \bar{q}_{1, k} \triangleq\left[q_{1}\left(k N_{1}\right), q_{1}\left(k N_{1}+1\right), \ldots, q_{1}\left((k+1) \cdot N_{1}-1\right)\right]
\end{aligned}
$$

where $k$ is the symbol index, $\bar{i}_{1}$ is the in-phase vector component and $\bar{q}_{1}$ is the quadrature vector component of the first chain. Then, $\bar{X}_{1}$ is defined as the DFT 1 output vector which includes up to $N_{1}$ complex values:

$$
\bar{X}_{1, k}=\operatorname{DFT}\left(\bar{i}_{1, k}+j \bar{q}_{1, k}, N_{1}\right)
$$

Similarly for the second chain using $\mathrm{DFT}_{2}$ with size $N_{2}$, we define:

$$
\bar{X}_{2, k}=\operatorname{DFT}\left(\bar{i}_{2, k}+j \bar{q}_{2, k}, N_{2}\right)
$$

Corresponding frequency bins are then multiplied:

$$
\bar{Y}_{k}=\bar{X}_{1, k} \odot\left(\bar{X}_{2, k}\right)^{*}
$$

where $\bar{Y}$ is the output of the multiplier, ${ }^{*}$ indicates the complex conjugate and $\odot$ indicates the element-wise multiplication. If integration is required to improve the performance of the receiver, the same symbol has to be repeated at the transmitter side $I$ times and the correlations of multiple (equal) symbols are summed:

$$
\bar{Z}_{n}=\frac{1}{I} \sum_{k=n \cdot I}^{(n+1) \cdot I-1} \bar{Y}_{k}
$$

where $\bar{Z}$ is the integrator output, $n$ is the index of the repeated symbol and $I$ is the number of integrations.

In order to use $\mathrm{XC}$ for communications instead of energy detection applications, FSK is used since it depends on the energy detected in different frequency channels. For FSK, detection is based on the comparison between the detected energy in the used frequency bins in the demodulator. In the

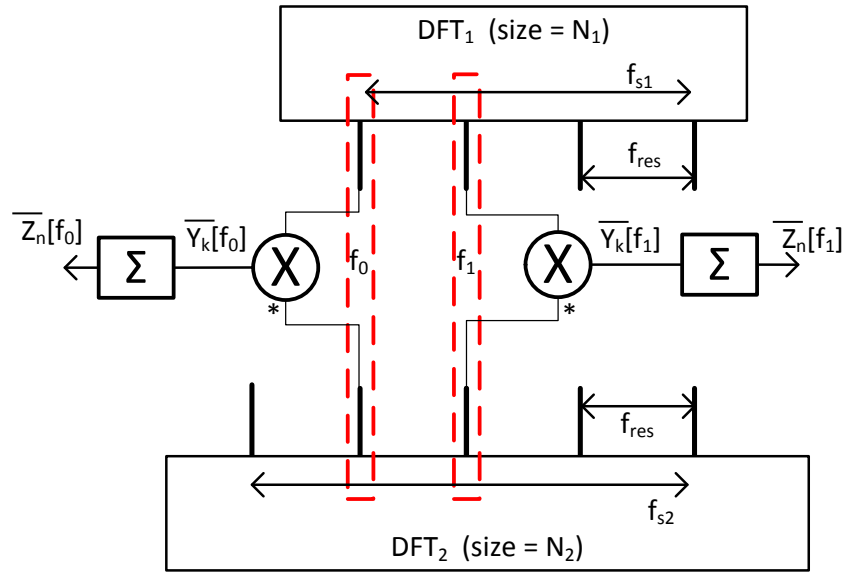

Fig. 4. A detailed view of the DFT in the cross-correlator.

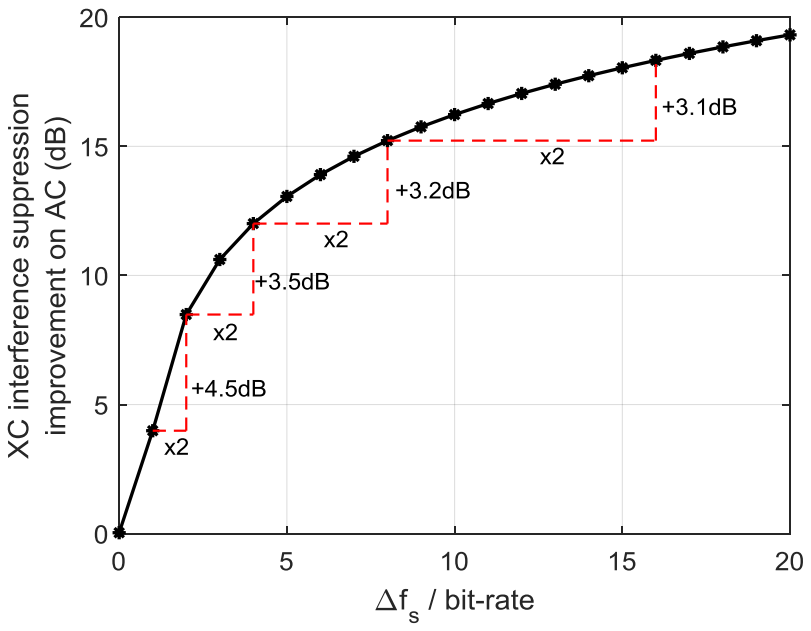

Fig. 5. The interference suppression (limited by spectral leakage only) of $\mathrm{XC}$ compared to $\mathrm{AC}$ versus the difference between the sampling frequencies of the two chains $\left(\Delta f_{s}\right)$. As $\Delta f_{s}$ increases, the suppression is higher.

following sections, BFSK was used to test the design. In this case, as shown in Fig. 4, only two outputs of the DFT are needed that correspond to $f_{0}$ and $f_{1}$, the two modulation frequencies used by BFSK. In this case, only the two bins that correspond to these two frequencies have to be calculated, so the processing can be much more efficient than calculating an N-point DFT. The XC calculates the energy in these two frequencies and the demodulator compares them to each other to decode the received bits:

$$
\begin{aligned}
& \overline{Z_{n}}\left[f_{0}\right] \geq \overline{Z_{n}}\left[f_{1}\right] \rightarrow \text { bit }=0 \\
& \overline{Z_{n}}\left[f_{0}\right]<\overline{Z_{n}}\left[f_{1}\right] \rightarrow \text { bit }=1
\end{aligned}
$$

\section{INTERFERENCE SUPPRESSION}

To estimate the interference suppression, we assume a sinusoidal interferer. Ideally, if the interference frequency is outside the band of correlation, it would have no effect after the XC since it will appear at different frequencies in both chains. In a real implementation, however, there are some limitations that will reduce the interference suppression such as 
multiplication with noise in the other receiver, spectral leakage and a reduced desired output, as will be explained next.

Due to the usage of rectangular windowing in the implementation of a limited-size DFT, the sinusoidal interference produces an output in all DFT bins due to spectral leakage (unless the interference happens to fall at specific frequencies of which the odds are virtually zero). Multiplying the DFT bins of both chains would then create non-zero output just due to the interference. This undesirable output is dependent on $\Delta f_{s}$, the difference between the sampling frequencies of both chains.

Fig. 5 shows the simulated interference suppression of only spectral leakage by $\mathrm{XC}$ at different $\Delta f_{s}$ compared to the $\mathrm{AC}$ output. In practice, the interference suppression will definitely be worse than this because of other factors such as noise. For $\Delta f_{s}=0$, in which interference aliasing is the same in both chains, the worst-case XC scenario yields the same interference performance as $\mathrm{AC}$, i.e. $0 \mathrm{~dB}$ difference. For higher $\Delta f_{s}$, the difference between the frequencies occupied by the interference after sub-sampling is larger. Thus the resulting interference power after correlation will be lower. In case where $\Delta f_{s}$ is 4 times the bit-rate, the interference power after $\mathrm{XC}$ is $12 \mathrm{~dB}$ lower than the $\mathrm{AC}$ output, while if $\Delta f_{s}$ is 8 times the bit-rate, this difference increases to about $15 \mathrm{~dB}$.

Another factor that degrades the interference suppression of the XC is what we refer to as the "reduced desired output". It happens when both the desired signal and an interference component appear in one of the chains at the same frequency bin after sub-sampling but with opposite phase and equal magnitude. Since these components add as vectors, they sum to zero and the DFT data output bin is nulled. Thus, the $\mathrm{XC}$ output is also nulled. The occurrence of this event will degrade the interference suppression performance in some specific cases as will be shown in section V.

\section{RESUlts}

In order to examine the performance of the proposed subsampling XC architecture, both Matlab simulations and an experimental setup were used. The setup includes a signal generator, a digital scope which includes both a sampler and a quantizer, and Matlab in which the sub-sampling and the digital baseband operations are performed.

\section{A. Noise suppression}

In order to measure noise suppression, white Gaussian noise is added to the signal. The BER of the output is then recorded, while varying the noise level, for both $\mathrm{AC}$-where only one receiver chain is used- and XC. BFSK modulation is used with $12.5 \mathrm{MHz}$ carrier frequency, $70 \mathrm{kbps}$ data-rate and $70 \mathrm{kHz}$ frequency spacing between the two frequencies used in the modulation. The sampling frequencies of the receivers are $1.19 \mathrm{MHz}$ and $1.47 \mathrm{MHz}$. The test was done using 5000 symbols to measure the BER. The noise bandwidth used is $100 \mathrm{MHz}$ and the noise folding due to different sampling frequencies will result in a low correlation coefficient between both chains. Fig. 6 shows the BER versus the signal to total noise ratio (SNR) at the input of the receiver. At a BER of $10^{-3}$, the figure shows $\sim 2 \mathrm{~dB}$ SNR improvement due to XC

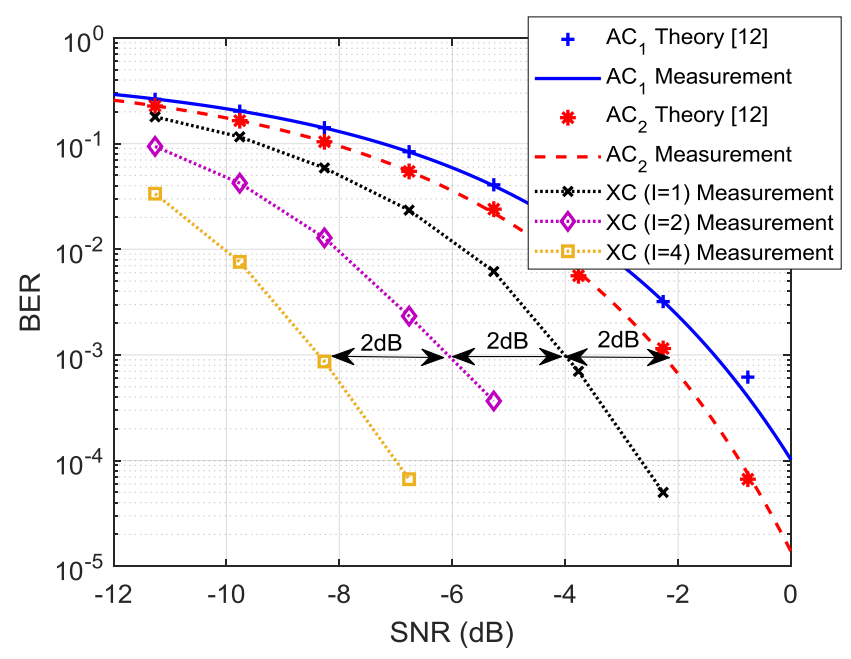

Fig. 6. Measured BER vs. SNR for AC and XC with 1, 2 and 4 integrations. SNR is measured at the reference point as indicated in Fig. 3.

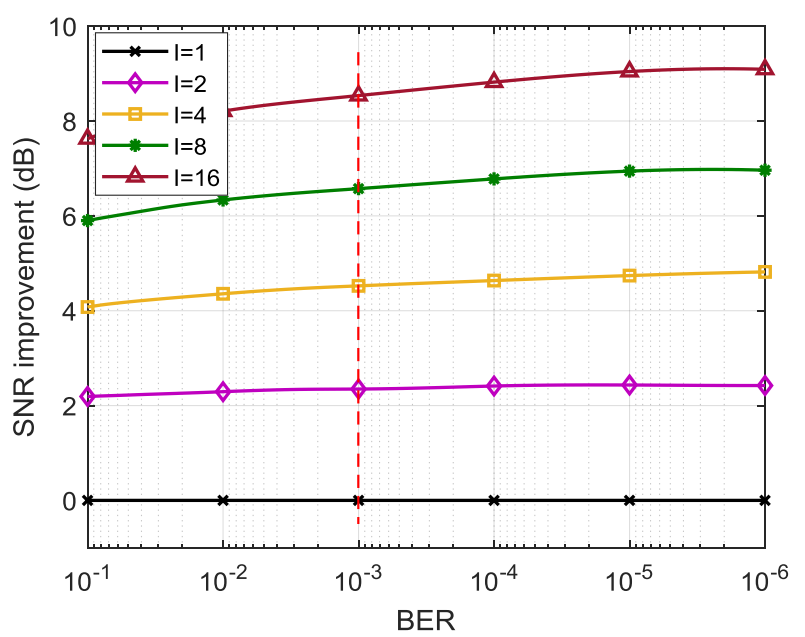

Fig. 7. Simulated SNR improvement due to increasing the number of integrations. Doubling the number of integrations improves SNR by $\sim 2 \mathrm{~dB}$.

compared to $\mathrm{AC}$, and an additional $\sim 2 \mathrm{~dB}$ for each doubling of integrations.

Fig. 7 shows the simulated SNR improvement for increasing the number of integrations. Doubling the number of integrations reduces the SNR required to achieve a specific BER by just over $2 \mathrm{~dB}$.

\section{B. Interference robustness}

In order to test the interference robustness of our architecture, a sinusoidal interferer in the band adjacent to the band of correlation was added to the BFSK signal. The same setup and frequencies discussed earlier in subsection V-A are used. The sinusoidal interferer frequency is chosen such that it falls at baseband exactly at the same frequency as the desired signal after sub-sampling for one of the chains. The signal-tointerference ratio (SIR) is then varied and the BER is recorded.

For the purpose of illustration, Fig. 8 shows the BER versus the SIR for AC (using only one chain) and XC with 1, 2 and 4 


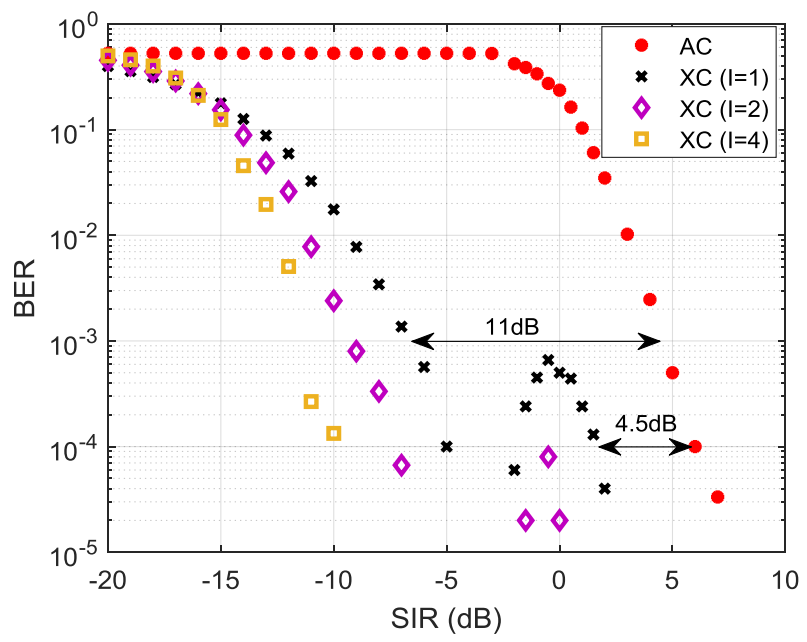

Fig. 8. Measured BER versus SIR for a sinusoidal interferer in case of $\mathrm{AC}$ and $\mathrm{XC}$ with 1, 2 and 4 integrations. The interferer frequency is chosen to fall at same frequency with an BFSK frequency after sub-sampling. Nonmonotonic behavior appears in the XC BER curve due to the reduced desired output effect.

integrations. In order to achieve a BER of $10^{-3}$, the required SIR is $4 \mathrm{~dB}$ and $-7 \mathrm{~dB}$ for $\mathrm{AC}$ and $\mathrm{XC}$ respectively which is an $11 \mathrm{~dB}$ improvement. However, for a BER of $10^{-4}$, the SIR improvement reduces to only $4.5 \mathrm{~dB}$. This can be explained by the reduced desired output power as discussed in section IV. As expected, this is most pronounced at $\mathrm{SIR} \approx 0 \mathrm{~dB}$ which shows as a local maximum in the curve. Moving leftward from SIR $=0 \mathrm{~dB}$, the interferer power increases yet the BER decreases due to a lower probability of the nulling effect.

More general conclusions can be drawn when varying the interference frequency within the band adjacent to the band of correlation and searching for the case where the maximum SIR is required to achieve a specific BER. For each interferer frequency with $7 \mathrm{kHz}$ spacing $\left(=0.1 f_{\text {res }}\right), 100$ tests are simulated where the desired signal includes 1000 bits and the sinusoidal interferer has a different random phase in each test. In order to summarize the results, we report the SIR required to guarantee a specific BER across the band adjacent to the band of correlation. Fig. 9 shows the BER versus the required SIR in case of $\mathrm{AC}$ and XC. In order to guarantee a BER of $10^{-3}$, the SIR should be at least $4.5 \mathrm{~dB}, 0 \mathrm{~dB},-7 \mathrm{~dB}$ and $-10 \mathrm{~dB}$ for $\mathrm{AC}$ and for $\mathrm{XC}$ with 1,2 and 4 integrations respectively. This leads to respective interference suppression of $4.5 \mathrm{~dB}, 11.5 \mathrm{~dB}$ and $14.5 \mathrm{~dB}$ improvement compared to the traditional AC solution.

\section{CONCLUSION}

Sub-sampling receivers use a digital-intensive architecture to save power in the analog front-end, but they suffer from noise folding and interference aliasing. Solutions were proposed in literature using RF-filters but they are bulky and/or power-hungry. In this paper, a solution is proposed by using two receiver chains and cross-correlating their outputs. In the proposed implementation, two different sub-sampling frequencies are used to reduce the effects of noise and interference aliasing thereby relaxing the specifications of the RF-filters. This allows the receiver to work in environments with low

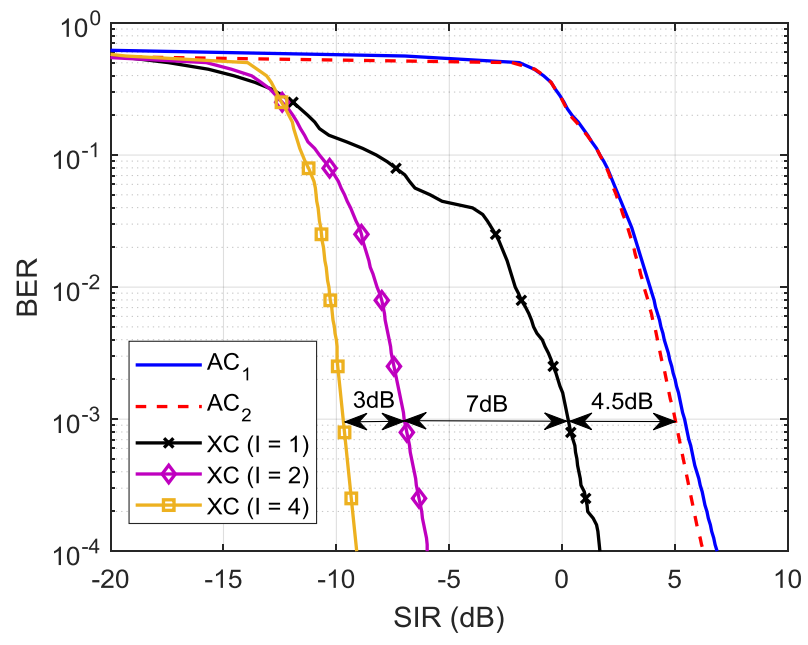

Fig. 9. Simulated SIR required to guarantee a specific BER for a sinusoidal interferer at any frequency in the band adjacent to the band of correlation.

SINR. Measurements on the proposed receiver show $\sim 2 \mathrm{~dB}$ SNR improvement due to $\mathrm{XC}$ and an additional $\sim 2 \mathrm{~dB}$ for each doubling of integrations. The required SIR is reduced significantly by $\mathrm{XC}$ as much as $14.5 \mathrm{~dB}$ using quarter the datarate.

\section{REFERENCES}

[1] K. Philips, "Ultra low power short range radios: Covering the last mile of the IoT," in 40th European Solid State Circuits Conference (ESSCIRC), Venice, Italy, Sept. 2014, pp. 51-58.

[2] R. G. Vaughan, N. L. Scott, and D. R. White, "The theory of bandpass sampling," IEEE Transactions on Signal Processing, vol. 39, no. 9, pp. 1973-1984, Sept. 1991.

[3] A. A. Abidi, "The path to the software-defined radio receiver," IEEE Journal of Solid-State Circuits, vol. 42, no. 5, pp. 954-966, May 2007.

[4] A. Heragu, D. Ruffieux, and C. C. Enz, "A 2.4-GHz MEMS-based PLLfree multi-channel receiver with channel filtering at RF," IEEE Journal of Solid-State Circuits, vol. 48, no. 7, pp. 1689-1700, July 2013.

[5] H. Pekau and J. W. Haslett, "A $2.4 \mathrm{GHz}$ CMOS sub-sampling mixer with integrated filtering," IEEE Journal of Solid-State Circuits, vol. 40, no. 11, pp. 2159-2166, Nov. 2005.

[6] J. Cheng, N. Qi, P. Y. Chiang, and A. Natarajan, "A low-power, low-voltage WBAN-compatible sub-sampling PSK receiver in $65 \mathrm{~nm}$ CMOS," IEEE Journal of Solid-State Circuits, vol. 49, no. 12, pp. 30183030, Dec. 2014.

[7] E. A. M. Klumperink, H. J. Westerveld, and B. Nauta, "N-path filters and mixer-first receivers: A review," in 2017 IEEE Custom Integrated Circuits Conference (CICC), Austin, TX, USA, Apr. 2017, pp. 1-8.

[8] M. S. Oude Alink, "RF spectrum sensing in CMOS exploiting crosscorrelation," Ph.D. dissertation, University of Twente, Enschede, May 2013.

[9] M. S. Oude Alink, E. A. M. Klumperink, A. B. J. Kokkeler, Z. Ru, W. Cheng, and B. Nauta, "Using cross-correlation to mitigate analog/RF impairments for integrated spectrum analyzers," IEEE Transactions on Microwave Theory and Techniques, vol. 61, no. 3, pp. 1327-1337, Mar. 2013.

[10] M. S. Oude Alink, A. B. J. Kokkeler, E. A. M. Klumperink, Z. Ru, W. Cheng, and B. Nauta, "Improving harmonic rejection for spectrum sensing using cross-correlation," in 38th European Solid State Circuits Conference (ESSCIRC), Bordeaux, France, Sept. 2012, pp. 361-364.

[11] Y. Zhao and S. Xiao, "Sparse multiband signal spectrum sensing with asynchronous coprime sampling," Cluster Computing, Feb. 2018. [Online]. Available: https://doi.org/10.1007/s10586-018-2297-6

[12] S. Haykin, Communication systems, 4th ed. Wiley Publishing, 2001. 